Pacific Journal of Mathematic 


\title{
THE BIGGER BRAUER GROUP AND ÉTALE COHOMOLOGY
}

\author{
IAIN RAEBURN AND JOSEPH L. TAYLOR
}

\begin{abstract}
The classical Brauer group $B(R)$ is formed from equivalence classes of Azumaya algebras over the ring $R$. The bigger Brauer group $\tilde{B}(R)$ is formed in a similar way from equivalence classes in a larger category of $R$-algebras. This larger category is defined through axioms similar to those defining Azumaya algebras but with the requirement for an identity dropped. In this paper we identify $\tilde{B}(R)$ with the second étale cohomology of $\operatorname{Spec}(R)$ (with $G_{m}$ as coefficients). The classical Brauer group consists of the torsion subgroup of this cohomology group. This result yields a concrete realization of second étale cohomology and also enables us to settle several questions about the relation of $\tilde{B}(R)$ to $H^{2}(\Delta, \mathbf{Z})$ in the case where $R$ is a Banach algebra with maximal ideal space $\Delta$.
\end{abstract}

That $B(R)$ may indeed be a proper subgroup of $\tilde{B}(R)$ is demonstrated by the fact that there is an isomorphism $\tilde{B}(R) \rightarrow H^{3}(\Delta, \mathbf{Z})$ if $R=C(\Delta)$ for a compact Hausdorff space $\Delta$ (cf. Prop. 6.6 of [12]). Since $B(R)$ is carried to the torsion subgroup of $H^{3}(\Delta, \mathbf{Z})$ by this map, $B(R)$ and $\tilde{B}(R)$ will be distinct if $H^{3}(\Delta, \mathbf{Z})$ is non-torsion. In the case $R=C(\Delta)$ the central separable algebras are related to another class of $R$ algebras - the algebras with continuous trace from $C^{*}$-algebra theory. In fact, in [11] we use an elementary proof of the surjectivity of the map $\tilde{B}(C(\Delta)) \rightarrow H^{3}(\Delta, \mathbf{Z})$ to give an elementary proof of the existence of continuous trace $C^{*}$-algebras of given Dixmier-Douady class (cf. [5]).

The map $\tilde{B}(R) \rightarrow H^{3}(\Delta, \mathbf{Z})$ is defined for any Banach algebra $R$ with maximal ideal space $\Delta$, but in general neither the injectivity nor surjectivity of this map was established in [12]. It was proved to be an injection on the sub-group $\bar{B}(R) \subset \tilde{B}(R)$ consisting of equivalence classes containing an algebra finitely presented as an $R$-module. The functor $\bar{B}$ agrees with $\tilde{B}$ on Noetherian rings and is continuous (commutes with direct limit) whereas $\tilde{B}$ was not proved to be continuous in [12]. In general, $B(R) \subset$ $\bar{B}(R) \subset \tilde{B}(R)$. However, the following questions were left unanswered in [12]: Is $\bar{B}(R)$ always equal to $\tilde{B}(R)$ ? Is $\bar{B}(R)$ always $B(R)$ ? (This would force $B(R)=\tilde{B}(R)$ in the Noetherian case - a possibility left open in [12].) When $R$ is a commutative Banach algebra with maximal ideal space $\Delta$, is $\tilde{B}(R) \rightarrow H^{3}(\Delta, \mathbf{Z})$ always surjective? Is it always injective? Is $\bar{B}(R)$ $\rightarrow H^{3}(\Delta, \mathbf{Z})$ always surjective? 
We can now answer these questions. The key is to realize that the correct cohomology group in which to embed $\tilde{B}(R)$ is the étale cohomology group $H^{2}\left(X_{\mathrm{et}}, \mathbf{G}_{m}\right)$ where $X=\operatorname{Spec}(R)$ (cf. [10]). This will come as no surprise to mathematicians familiar with Grothendieck's work on étale cohomology and the Brauer group (it might have occurred much earlier to the author of [12] had he known any étale cohomology). The classical Brauer group $B(R)$ has a natural injection into $H^{2}\left(X_{\mathrm{et}}, \mathbf{G}_{m}\right)$ ([7], [10]), but the image is always torsion, while $H^{2}\left(X_{\mathrm{et}}, \mathbf{G}_{m}\right)$ is not always torsion. Thus, in $\tilde{B}$ and $H^{2}\left(\cdot, \mathbf{G}_{m}\right)$ we have two functors that behave like bigger Brauer groups and it is natural to ask if they agree. In fact, we shall prove that for $X=\operatorname{Spec}(R)$ there is always an isomorphism $\tilde{B}(R) \rightarrow$ $H^{2}\left(X_{\text {et }}, \mathbf{G}_{m}\right)$.

With the above result it becomes easy to answer the questions left over from [12]: For any ring $R$, we have $\bar{B}(R)=\tilde{B}(R) \simeq$ $H^{2}\left(\operatorname{Spec}(R)_{\mathrm{et}}, \mathbf{G}_{m}\right)$. Thus, $\tilde{B}(R) \rightarrow H^{3}(\Delta, \mathbf{Z})$ is injective if $R$ is a Banach algebra with maximal ideal space $\Delta$. However, this map need not be surjective (cf. §4) because, roughly speaking, the étale topology is often not rich enough to allow other than torsion elements of $H^{3}(\Delta, \mathbf{Z})$ to be represented by cocycles in the étale topology. Thus, $\tilde{B}$ is not the functor the second author was seeking when he wrote [12] (cf. $\$ 7$ of [12]). However, it may be of interest in commutative algebra as a concrete realization of $H^{2}\left(X_{\mathrm{et}}, \mathbf{G}_{m}\right)$.

Since the tools used here are those of schemes and étale cohomology, it is not much extra effort to extend the definition of $\tilde{B}$ to schemes and to prove a version of the theorem relating it to étale cohomology in this context.

We introduce $\tilde{B}$ for schemes $X$ in $\$ 1$. In $\$ 2$ we define an injection $\delta: \tilde{B}(X) \rightarrow H^{2}\left(X_{\text {et }}, \mathbf{G}_{m}\right)$ following Giraud [7]. We also, in $\$ 2$, use localization techniques to settle another question left over from [12]: we prove the triviality of the module $\Omega(A)$ of $\S 3$ of [12]. In $\S 3$ we prove that $\delta$ is surjective for schemes which satisfy the hypotheses of Artin's Lemma [1]. Using Grothendieck's continuity results, we extend this result to affine schemes in \$4. With the help of standard results about étale cohomology we then settle the questions raised in [12].

We would like to thank Gus Lehrer of Sydney University for his guidance concerning the literature on étale cohomology.

1. The bigger Brauer group of a scheme. By the term ring we shall mean commutative ring with identity. However, algebras need not be commutative or have identities. 
If $A$ is an algebra over a ring $R$, then $A$ is separable in the sense of [12] if

(i) $A^{2}=A$;

(ii) for each maximal ideal $M \subset R, M A \neq A$;

(iii) $A$ is a projective $A$-bimodule.

Note that (i) and (ii) are redundant if $A$ has an identity.

We denote the algebra $\operatorname{End}_{A-A}(A)$ by $\mathbf{Z}(A)$. If the natural map $R \rightarrow \mathbf{Z}(A)$ is an isomorphism, we say $A$ is central. A central separable algebra is one which is both central and separable. If such an algebra also has an identity, then we shall call it an Azumaya algebra.

The classical Brauer group $B(R)$ consists of Morita equivalence classes of Azumaya algebras with tensor product as operation. The bigger Brauer group $\tilde{B}(R)$, introduced in [12], consists of Morita equivalence classes of central separable $R$-algebras under tensor product. The identity class in $B(R)$ consists of all algebras of the form $\operatorname{End}_{R}(M)$, where $M$ is a finitely generated projective $R$-module. The identity class in $\tilde{B}(R)$ consists of all algebras of the form $M \otimes_{R}^{\lambda} N$, where $M$ and $N$ are $R$-modules, $\lambda: N \otimes_{R} M \rightarrow R$ is an $R$-module surjection, and $M \otimes_{R}^{\lambda} N$ is $M \otimes_{R} N$ with multiplication defined by $\lambda$ through the formula

$$
\left(m_{1} \otimes n_{1}\right) \cdot\left(m_{2} \otimes n_{2}\right)=\lambda\left(n_{1} \otimes m_{2}\right)\left(m_{1} \otimes n_{2}\right) ;
$$

we call this the elementary $R$-algebra determined by the data $(M, N, \lambda)$. Note that if $M$ is finitely generated and projective, $N=M^{\prime}=$ $\operatorname{Hom}_{R}(M, R)$, and $\lambda$ is the standard pairing $M \otimes_{R} M^{\prime} \rightarrow R$, then $M \otimes_{R}^{\lambda} N$ is just $\operatorname{End}_{R}(M)$. Note also, by Proposition 4.6 of [12] there is a canonical injection $B(R) \rightarrow \tilde{B}(R)$.

We now describe a useful alternative characterization of central separable algebras given in [12]. Let $A$ be an $R$-algebra and set $A^{e}=A \otimes_{R} A^{\text {op }}$. Following $\S 3$ of [12], we let $A_{1}$ be $A$ with the left $A^{e}$-module structure given by $(a \otimes b) \cdot c=a c b$, while $A_{\mathrm{r}}$ is $A$ with the right $A^{e}$-module structure given by $c \cdot(a \otimes b)=b c a$. Then $A^{e}$ and $A^{p}=A_{1} \otimes_{R} A_{\mathrm{r}}$ are two distinct $A^{e}$-bimodules, although both have $A \otimes_{R} A$ as underlying $R$-module. We set

$$
\Omega(A)=\operatorname{Hom}_{A^{e}-A^{e}}\left(A^{p}, A^{e}\right),
$$

to heredefine $\theta: A^{e} \otimes_{R} \Omega(A) \rightarrow A^{p}$ by

$$
\theta(\alpha \otimes \omega)=\omega(\alpha),
$$

and define tr: $A \otimes_{R} \Omega(A) \rightarrow \mathbf{Z}(A)$ by

$$
\operatorname{tr}(a \otimes \omega) b=\pi \circ \omega(b \otimes a)=\pi \circ(b \otimes a \otimes \omega)
$$

where $\pi: A^{e} \rightarrow A$ is the multiplication map. 
We restate Proposition 3.7 and 3.8 of [12] as follows:

Proposition 1.1. Let $A$ be a faithful R-algebra. If there is an $R$-submodule $\Omega^{\prime} \subset \Omega(A)$ so that tr maps $A \otimes_{R} \Omega^{\prime}$ onto $R \subset \mathbf{Z}(A)$, then $A$ is central separable.

Conversely, if $A$ is central separable, then

(a) $\operatorname{tr}: A \otimes_{R} \Omega(A) \rightarrow R$ is surjective;

(b) $\theta: A^{p} \otimes_{R} \Omega(A) \rightarrow A^{e}$ is an isomorphism; and

(c) $\Omega(A)$ is an invertible $R$-module.

Actually, $\Omega(A) \simeq R$, so that tr yields a surjective trace $A \rightarrow R$ and $\theta$ an isomorphism $A^{p} \rightarrow A^{e}$. This was conjectured in [12]. We can now prove it using localization techniques (cf. Proposition 2.2).

The following was used implicitly throughout $\S 4$ of [12], but should be made explicit:

COROLlaRY 1.2. If $R \rightarrow S$ is a ring homomorphism (preserving identities) and $A$ is a central separable $R$-algebra, then $A \otimes_{R} S$ is a central separable $S$-algebra.

Proof. For $\omega \in \Omega(A)$ the map $\omega_{S}=\omega \otimes$ id: $A^{p} \otimes_{R} S \rightarrow A^{e} \otimes_{R} S$ belongs to $\left(\Omega(A) \otimes_{R} S\right)$. If $\Omega^{\prime} \subset \Omega\left(A \times{ }_{R} S\right)$ is the $S$-module spanned by all such elements, then by Proposition 1.1 (a), tr: $\left(A \otimes_{R} S\right) \otimes_{R} \Omega^{\prime} \Rightarrow S$ is surjective and, hence, $A \otimes_{R} S$ is a central separable $S$-algebra.

We shall now define $\tilde{B}(X)$ for schemes $X$, following the analogous definition for the classical Brauer group (cf. [10], IV). For terminology and notation on schemes we follow Hartshorne [9].

Definition 1.3. Let $X$ be a scheme with structure sheaf $\mathcal{O}_{X}$. A quasi-coherent sheaf $\mathscr{A}$ of $\mathcal{O}_{X}$-algebras will be called a central separable $\mathcal{O}_{X}$-algebra if for each $x \in X$ there is an affine neighborhood $U$ of $x$ such that $\Gamma(U, \mathscr{A})$ is a central separable $\Gamma\left(U, \mathcal{O}_{X}\right)$ algebra.

Note that by Corollary 1.2, if $A$ is any central separable $R$-algebra and $X=\operatorname{Spec} R$, then $A \otimes_{R} \mathcal{O}_{X}$ is a central separable $\mathcal{O}_{X}$ algebra. Conversely, given a scheme $X$ and a central separable $\mathcal{O}_{X}$-algebra $\mathscr{A}$, then $\Gamma(U, \mathscr{A})$ is a central separable $\Gamma\left(U, \mathcal{O}_{X}\right)$ algebra for each affine open set $U \subset X$. To see this we use Proposition 1.1. The modules $\Omega(A)$, as $A$ ranges over the algebras $\Gamma(U, \mathscr{A})$, form a presheaf which satisfies the conditions to be the pre-sheaf of sections of a sheaf $\Omega$. Then $\operatorname{tr}$ defines a sheaf homomorphism $\mathscr{A} \otimes \Omega \rightarrow \mathcal{O}_{X}$ which is surjective by Proposition 1.1 , since $\mathscr{A}$ is locally central separable. On passing to sections over an 
affine open set $U$ and using quasi-coherence (cf. [9], II, 5.2, 5.6, 5.7) we conclude that

$$
\operatorname{tr}: A \otimes_{R} \Omega(A) \rightarrow R
$$

is surjective with $R=\Gamma\left(U, \mathcal{O}_{X}\right), A=\Gamma(U, \mathscr{A})$, and $U$ any affine open set. Another application of Proposition 1.1 shows that $\Gamma(U, \mathscr{A})$ is central separable over $\Gamma\left(U, \mathcal{O}_{X}\right)$. In particular, it is easy to see that if $X=\operatorname{Spec} R$ for a ring $R$, then $\mathscr{A} \rightarrow \Gamma(X, \mathscr{A})$ is an equivalence between the category of central separable $\mathcal{O}_{X}$ algebras and the category of central separable $R$-algebras.

We are indebted to A. Verschoren for pointing out that we cannot get away with simple insisting that each stalk $\mathscr{A}_{x}$ be central separable in Definition 1.3 unless we assume that $\mathscr{A}^{p}$ is locally a finitely presented $\mathscr{A}^{e}$-module. We made this mistake in an earlier version of this paper.

Henceforth, if we are working over a scheme $X$ the symbol $\otimes$ will refer to tensor product relative to the structure sheaf $\mathcal{O}_{X}$.

We shall say that central separable $\mathscr{O}_{X}$-algebras $\mathscr{A}$ and $\mathscr{B}$ are equivalent if there exist quasi-coherent $\mathcal{O}_{X}$-sheaves $\mathscr{M}$ and $\mathscr{N}$ such that $\mathscr{M}$ is a regular $\mathscr{A}-\mathscr{B}$ bimodule, $\mathscr{N}$ is a regular $\mathscr{B}-\mathscr{A}$ bimodule, $\mathscr{M} \otimes_{\mathscr{B}} \mathscr{N} \simeq \mathscr{A}$ as an $\mathscr{A}$ bimodule, and $\mathscr{N} \otimes_{\mathscr{A}} \mathscr{M} \simeq \mathscr{B}$ as a $\mathscr{B}$ bimodule (cf. [12], §4). Here, the term regular applied to $\mathscr{M}$ and $\mathscr{N}$ means $\mathscr{A} \otimes_{\mathscr{A}} \mathscr{M} \otimes_{\mathscr{B}} \mathscr{B}=\mathscr{M}$ and $\mathscr{B} \otimes_{\mathscr{B}} \mathscr{N} \otimes_{\mathscr{A}} \mathscr{A}=\mathscr{N}$.

Now suppose $\mathscr{B}$ is a central separable $\mathcal{O}_{X}$-algebra, $\mathscr{M}$ is a regular $\left(\mathscr{M} \otimes_{\mathscr{B}} \mathscr{B}=\mathscr{M}\right)$ quasi-coherent right $\mathscr{B}$-mdoule, $\mathscr{N}$ is a regular quasicoherent left $\mathscr{B}$-module, and $\lambda: \mathscr{N} \otimes \mathscr{M} \rightarrow \mathscr{B}$ is a surjective $\mathscr{B}$ - $\mathscr{B}$ bimodule homomorphism. Then one proves as in $\S 4$ of [12] that the quasi-coherent $\mathcal{O}_{X}$-algebra $\mathscr{A}=\mathscr{M} \otimes_{\mathscr{\mathscr { C }}}^{\lambda} \mathscr{N}$, with product given by

$$
1 \otimes \lambda \otimes 1: \mathscr{M} \otimes_{\mathscr{B}} \mathscr{N} \otimes \mathscr{M} \otimes_{\mathscr{B}} \mathscr{N} \rightarrow \mathscr{M} \otimes_{\mathscr{B}} \mathscr{B} \otimes_{\mathscr{B}} \mathscr{N}=\mathscr{M} \otimes_{\mathscr{B}} \mathscr{N}
$$

is a central separable $\mathcal{O}_{X}$ algebra and is equivalent to $\mathscr{B}$. Furthermore, every central separable $\mathcal{O}_{X}$ algebra equivalent to $\mathscr{B}$ arises in this way. In particular, the central separable $\mathcal{O}_{X}$-algebras equivalent to $\mathcal{O}_{X}$ itself are given as above with $\mathscr{B}=\mathcal{O}_{X}$. We call such an algebra the elementary $\mathcal{O}_{X}$-algebra determined by the data $(\mathscr{M}, \mathscr{N}, \lambda)$.

Tensor products of central separable algebras over a ring $R$ are again central separable (cf. [12], Prop. 2.7) so the same is clearly true over a scheme. Also, if $R \rightarrow S$ is a ring homomorphism and $A$ a central separable $R$-algebra then $A \otimes_{R} S$ is, by Corollary 1.2, a central separable $S$-algebra. The analogous statement for schemes is that if $\mathscr{A}$ is a central separable $\mathcal{O}_{X}$ algebra and $f: Y \rightarrow X$ is a morphism of schemes, then $f^{*} \mathscr{A}$ is a central separable $\mathcal{O}_{Y}$-algebra ([9], II. §5). 
Definition 1.4. If $X$ is a scheme, we denote by $\tilde{B}(X)$ the group under tensor product, of equivalent classes of central separable $\mathcal{O}_{X}$-algebras (cf. [12], §4).

The identity in $\tilde{B}(X)$ is the class of all elementary $\mathcal{O}_{X}$ algebras. For $\mathscr{A}$ central separable, the inverse of $[\mathscr{A}] \in \tilde{B}(X)$ is $\left[\mathscr{A}^{\text {op }}\right]$ (cf. Proposition 3.10 of [12]). That $\tilde{B}$ is a functor from schemes to abelian groups follows from the comment preceding the definition.

Again proceeding as in $\S 4$ of [12], one can prove that the classical Brauer group $B(X)$ of a scheme $X$ is contained in $\tilde{B}(X)$. It is the subgroup consisting of equivalence classes which contain a central separable algebra $\mathscr{A}$ for which each $\mathscr{A}_{x}$ is an Azumaya algebra (has an identity).

2. Étale cohomology. We shall use Milne [10] as our main reference for étale cohomology.

Let $\mathbf{G}_{m}$ denote the sheaf on the étale site over $X$ which has $\Gamma\left(U, \mathcal{O}_{U}^{*}\right)$ as its group of sections over $U \rightarrow X$ ([10], II.1). Then for any scheme $X$ there is an injective morphism $B(X) \rightarrow H^{2}\left(X_{\mathrm{et}}, \mathbf{G}_{m}\right)$ ([10], IV. 2.5). The same thing is true with $B$ replaced by $\tilde{B}$ and, in fact, the proofs are nearly identical once one establishes appropriate localizing lemmas.

LEMMA 2.1. Let $X$ be a scheme and $\mathscr{A}$ a central separable $\mathcal{O}_{X}$ algebra. Then each point of $X$ is covered by an étale morphism of finite type $f$ : $U \rightarrow X$ such that $U$ is affine, $\Gamma\left(U, f^{*} \mathscr{A}\right)$ contains a rank one idempotent, and $f^{*} \mathscr{A}$ is an elementary $\mathcal{O}_{U^{-}}$algebra.

Proof. For $x \in X$ let $\bar{x}$ be a geometric point over $x$ and consider $\boldsymbol{\theta}_{X, \bar{x}}=\lim _{\boldsymbol{\alpha}} \Gamma\left(U_{\alpha}, \mathcal{O}_{U}\right)$, where the limit is over all étale maps $f_{\alpha}: U_{\alpha} \rightarrow X$, $U_{\alpha}$ affine, through which $\bar{x} \rightarrow x$ factors ([10], I. 4.11). Since $\mathcal{O}_{X, \bar{x}}$ is a Henselian local ring with algebraically closed residual field, Proposition 4.9 of [12] implies that $\lim _{\leftarrow} \Gamma\left(U_{\alpha}, f_{\alpha}^{*} \mathscr{A}\right)$ contains a rank one idempotent, and, hence, that, for some $\alpha, \Gamma\left(U_{\alpha}, f_{\alpha}^{*} \mathscr{A}\right)$ contains a rank one idempotent. By restricting to a smaller open affine set if necessary, we may assume $f_{\alpha}: U_{\alpha} \rightarrow X$ has finite type. By Proposition 4.3 of [12], $\Gamma\left(U_{\alpha}, f_{\alpha}^{*} \mathscr{A}\right)$ is elementary and, hence, $f_{\alpha}^{*} \mathscr{A}$ is as well since $U_{\alpha}$ is affine (cf. [9], II. 5.2, 5.5).

At this point we digress in order to prove the triviality of the module $\Omega(\mathscr{A})$.

Proposition 2.2. If $\mathscr{A}$ is a central separable $\mathcal{O}_{X}$-algebra, then there is a natural isomorphism $\Omega(\mathscr{A}) \rightarrow \mathcal{O}_{X}$. Hence, there is an isomorphism $\mathscr{A}^{p} \rightarrow \mathscr{A}^{e}$ and a surjective trace $\operatorname{tr}: \mathscr{A} \rightarrow \mathcal{O}_{X}$. 
Proof. First, suppose $R$ is a ring and $A$ a central separable $R$-algebra containing a rank one idempotent $p$. By definition ([12], §4), $p A p=R p$. This implies that $\Theta: A^{p} \otimes_{R} \Omega(A) \rightarrow A^{e}$ maps $p \otimes p \otimes \Omega(A)$ onto $R(p \otimes p)$ and, hence, is given by an isomorphism $\nu: \Omega(A) \rightarrow R$. In fact, from the definition of trace one sees that $\nu(\omega)=\operatorname{tr}(p \otimes \omega)$.

It turns out that $\nu$ is independent of the choice of $p$. To see this, let $q$ be another rank one idempotent in $A$ and, for each $\omega$, consider $\theta(q \otimes p \otimes \omega) \in q A p \otimes_{R} p A q$. Since $\pi \circ \theta(q \otimes p \otimes \omega)=\operatorname{tr}(p \otimes \omega) q=$ $\nu(\omega) q$, we conclude that $(q A p)(p A q)=R q$. If $R$ is a local ring with maximal ideal $M$, we may choose $u_{1} \in q A p, v \in p A q$ with $u_{1} v=r q$ and $r \notin M$. Then $u=r^{-1} u_{1}$ satisfies $u v=q$ and since $v u$ is a non-zero idempotent in $R p$ it has no choice but to be $p$. Then $\operatorname{tr}(q \otimes \omega)=\operatorname{tr}(u v \otimes \omega)$ $=\operatorname{tr}(v u \otimes \omega)=\operatorname{tr}(p \otimes \omega)$ by Proposition 3.6 of [12]. That this holds for local rings means, that for general $R, \operatorname{tr}(p \otimes \omega)$ and $\operatorname{tr}(q \otimes \omega)$ will have equal images in each local ring $R_{x}$ for $x \in \operatorname{Spec}(R)$ and, hence, will be equal. It follows that $\nu$ is independent of $p$.

Now let $X$ be a scheme and $\mathscr{A}$ any central separable $\mathcal{O}_{X}$ algebra. Choose a cover $\left\{f_{i}: U_{i} \rightarrow X\right\}$ be étale maps as in Lemma 2.1. Thus, $U_{i}$ is affine and $\Gamma\left(U_{i}, f_{i}{ }^{*} \mathscr{A}\right)$ contains a rank one idempotent. Then for each $i$, we have a canonical isomorphism $\nu_{i}: \Omega\left(f_{i}^{*} \mathscr{A}\right) \rightarrow \mathcal{O}_{U_{i}}$. Clearly, $\Omega\left(f_{i}^{*} \mathscr{A}\right)=$ $f_{i}^{*} \Omega(\mathscr{A})$ and, by the independence of $\nu$, the liftings $p_{1}^{*} \nu_{i}$ and $p_{2}^{*} \nu_{j}$ of $\nu_{i}$ and $\nu_{j}$ to the fiber product $U_{i} \times{ }_{X} U_{j}$ agree for each $i$ and $j$. It follows that there is an isomorphism $\nu: \Omega(\mathscr{A}) \rightarrow \mathcal{O}_{X}$ such that each $\nu_{i}$ is the lift of $\nu$ to $U_{i}$ (cf. [10], II. 1.6).

We return to the task of proving that $\tilde{B}(X)$ has an injection into $H^{2}\left(X_{\mathrm{et}}, \mathbf{G}_{m}\right)$. This will follow easily once we have two more localization lemmas.

LEMMA 2.3. If $X$ is a scheme and $\mathscr{A}=\mathscr{M}_{1} \otimes^{\lambda_{1}} \mathscr{N}_{1}$ and $\mathscr{M}_{2} \otimes^{\lambda_{2}} \mathscr{N}_{2}$ are isomorphic elementary $\mathscr{A}$-algebras over $X$, then there exists an invertible $\mathcal{O}_{X}$-module $\mathscr{P}$ such that $\mathscr{M}_{2} \simeq \mathscr{P} \otimes_{\mathscr{O}} \mathscr{M}_{1}, \mathscr{N}_{2} \simeq \mathscr{P}^{-1} \otimes_{\mathcal{O}} \mathscr{N}_{1}$, and $\lambda_{2}$ is $\lambda_{1}$ composed with $\mathscr{N}_{2} \otimes_{\mathscr{O}} \mathscr{M}_{2} \rightarrow\left(\mathscr{P}^{-1} \otimes \mathscr{N}_{1}\right) \otimes\left(\mathscr{P} \otimes \mathscr{M}_{1}\right) \simeq \mathscr{N}_{1} \otimes \mathscr{M}_{1}$.

Proof. Note that $\mathscr{M}_{i}$ is a left $\mathscr{A}$-module and $\mathscr{N}_{i}$ a right $\mathscr{A}$-module under the action determined by $\lambda_{i}$. Also,

$$
\begin{aligned}
& \mathscr{M}_{2} \simeq \mathscr{A} \otimes_{A} \mathscr{M}_{2}=\left(\mathscr{M}_{1} \otimes \mathscr{N}_{1}\right) \otimes_{\mathscr{A}} \mathscr{M}_{2} \simeq \mathscr{M}_{1} \otimes\left(\mathscr{N}_{1} \otimes_{\mathscr{A}} \mathscr{M}_{2}\right) \\
& \mathscr{N}_{2} \simeq \mathscr{N}_{2} \otimes_{\mathscr{A}} \mathscr{A}=\mathscr{N}_{2} \otimes_{\mathscr{A}}\left(\mathscr{M}_{1} \otimes \mathscr{N}_{1}\right) \simeq\left(\mathscr{N}_{2} \otimes_{\mathscr{A}} \mathscr{M}_{1}\right) \otimes \mathscr{N}_{1} .
\end{aligned}
$$

We set $\mathscr{P}=\mathscr{N}_{1} \otimes_{\mathscr{A}} \mathscr{M}_{2}$ and prove, as in Proposition 5.5 of [12], that it is an invertible $\mathcal{O}_{X}$-module with inverse $\mathscr{N}_{2} \otimes_{\mathscr{A}} \mathscr{M}_{1}$. 
Sets of data $(\mathscr{M}, \mathcal{N}, \lambda)$ defining elementary algebras over $X$, are the objects in a category in which the morphisms $(\mu, \nu):\left(\mathscr{M}_{2}, \mathscr{N}_{2}, \lambda_{2}\right) \rightarrow$ $\left(\mathscr{M}_{1}, \mathscr{N}_{1}, \lambda_{1}\right)$ are pairs of module isomorphisms $\mu: \mathscr{M}_{2} \rightarrow \mathscr{M}_{1}, \nu: \mathscr{N}_{2} \rightarrow$ $\mathscr{N}_{1}$, with $\lambda_{2}=\lambda_{1} \circ(\nu \otimes \mu)$. Since invertible $\mathcal{O}_{X}$ modules are locally trivial in the Zarisky topology ([8], II. ex 5.7), the above lemma says that sets of data which define isomorphic elementary algebras are locally isomorphic.

If $(\mu, \nu)$ is an automorphism of a given set of data $(\mathscr{M}, \mathscr{N}, \lambda)$ for an elementary $\mathcal{O}_{X}$-algebra, then $\mu \otimes \nu$ determines an automorphism of the resulting algebra $\mathscr{M} \otimes^{\lambda} \mathscr{N}$. Thus, there is a morphism $\operatorname{Aut}(\mathscr{M}, \mathscr{N}, \lambda) \rightarrow$ $\operatorname{Aut}\left(\mathscr{M} \otimes^{\lambda} \mathscr{N}\right)$. As in Proposition 5.6 of [12] one shows easily that its kernel consists of the automorphisms $(\mu, \nu)$ of the form $\left(h, h^{-1}\right)$ for $h \in \Gamma\left(X, \mathbf{G}_{m}\right)$. Thus,

\section{LEMMA 2.4. There is an exact sequence}

$$
0 \rightarrow \Gamma\left(X, \mathbf{G}_{m}\right) \rightarrow \operatorname{Aut}(\mathscr{M}, \mathscr{N}, \lambda) \rightarrow \operatorname{Aut}\left(\mathscr{M} \otimes^{\lambda} \mathscr{N}\right)
$$

for each set $(\mathscr{M}, \mathcal{N}, \lambda)$ of data for an elementary $\mathcal{O}_{X}$-algebra.

Now let $\mathscr{A}$ be a central separable $\mathcal{O}_{X}$-algebra for a scheme $X$. For each étale map $f: U \rightarrow X$ set $\mathscr{A}_{U}=f^{*} \mathscr{A}$ and let $F_{\mathscr{A}}(U)$ be the category of all tuples $(\mathscr{M}, \mathscr{N}, \lambda: \alpha)$, where $(\mathscr{M}, \mathscr{N}, \lambda)$ is data for an elementary $\mathcal{O}_{U}$-algebra and $\alpha: \mathscr{M} \otimes_{\mathscr{O}_{U}}^{\lambda} \mathscr{N} \rightarrow \mathscr{A}_{U}$ is an isomorphism of $\mathcal{O}_{U}$-algebras. A morphism between elements of $F_{\mathscr{A}}(U)$ will be an isomorphism $\left(\mathscr{M}_{1}, \mathscr{N}_{1}, \lambda_{1}\right) \rightarrow\left(\mathscr{M}_{2}, \mathscr{N}_{2}, \lambda_{2}\right)$ for which the diagram

$$
\begin{aligned}
& \mathscr{M}_{1} \otimes_{\mathscr{O}_{U}}^{\lambda_{1}} \mathscr{N}_{1} \quad \rightarrow \quad \mathscr{M}_{2} \otimes_{\mathscr{O}_{U}}^{\lambda_{2}} \mathscr{N}_{2} \\
& \alpha_{1} \searrow \mathscr{A}_{U} \swarrow \alpha_{2}
\end{aligned}
$$

commutes. Then Lemmas 2.3 and 2.4 imply that $U \rightarrow F_{\mathscr{A}}(U)$ is a Gerbe bounded by $\mathbf{G}_{m}$ in the sense of Giraud [7] (also see [10], IV. 2.5). It is the trivial Gerbe exactly when $F_{\mathscr{A}}(X)$ is non-empty, i.e., exactly when $[\mathscr{A}]=0$ in $\tilde{B}(X)$. It follows that we have an injection $\tilde{B}(X) \rightarrow H_{g}^{2}\left(X, \mathbf{G}_{m}\right)$, where $H_{g}^{2}\left(X, \mathbf{G}_{m}\right)$ in the group defined in IV. 3.1.1 of [7]. However, $H_{g}^{2}\left(X, \mathbf{G}_{m}\right)$ $\simeq H^{2}\left(X_{\mathrm{et}}, \mathbf{G}_{m}\right)$ by IV.3.4 of [7]. Thus, we have

Proposition 2.5. If $X$ is a scheme, there is an injection $\delta: \tilde{B}(X) \rightarrow$ $H^{2}\left(X_{\mathrm{et}}, \mathbf{G}_{m}\right)$.

The proof given for this proposition is rather unsatisfying for the following reason: in the next section, in addressing surjectivity for $\delta$, we will need to use Čech cohomology and rather explicit constructions involving Čech cocycles. For this reason, we present an alternative proof 
of Proposition 2.5 in the case where derived functor and Čech cohomology agree for the étale site.

Let $X$ be a Noetherian scheme in which each finite set is contained in an affine open set. Then Artin's theorem [1] says that Čech and derived functor étale cohomology agree (cf. [10], III.2.17).

Now let $\mathscr{A}$ be a central separable $\mathcal{O}_{X}$-algebra. Since $X$ is quasi-compact, we can cover $X$ with finitely many étale maps as in Lemma 2.1. By taking disjoint union, we come up with a single étale morphism of finite type $f: U \rightarrow X$ such that $f$ is a cover and $f^{*} \mathscr{A}$ is isomorphic to an elementary $\mathcal{O}_{U^{-a l g e b r a}} \mathscr{M} \otimes^{\lambda} \mathscr{N}$. Let $U \times U \rightarrow X$ be the fiber product over $X$ of $f$ with itself and let $p_{i}: U \times U \rightarrow U$ be given by $p_{i}\left(u_{1}, u_{2}\right)=u_{i}$. Then, $\mathscr{A}_{1}=p_{1}^{*} f^{*} \mathscr{A}$ and $\mathscr{A}_{2}=p_{2}^{*} f^{*} \mathscr{A}$ are naturally isomorphic $\mathcal{O}_{U \times U}$ algebras and if $\mathscr{M}_{i}=p_{i}^{*} \mathscr{M}, \mathscr{N}_{i}=p_{i}^{*} \mathscr{N}, \lambda_{i}=p_{i}^{*} \lambda$ then $\mathscr{A}_{1} \simeq \mathscr{M}_{1} \otimes^{\lambda_{1}} \mathscr{N}_{1}$ and $\mathscr{A}_{2} \simeq \mathscr{M}_{2} \otimes^{\lambda_{2}} \mathscr{N}_{2}$.

Now the sets of data $\left(\mathscr{M}_{i}, \mathscr{N}_{i}, \lambda_{i}\right)$ may not be isomorphic over $U \times U$; however, they are locally isomorphic by the remark following Lemma 2.3 and, hence, lift to isomorphic sets of data on $W$ for some étale cover $W \rightarrow U \times U$. By Artin's Lemma ([1] or [10], III.2.18), there is an étale morphism of finite type $U_{1} \rightarrow U$ so that $U_{1} \times U_{1} \rightarrow U \times U$ factors through $W \rightarrow U \times U$. Thus, we may as well assume that the sets of data $\left(\mathscr{M}_{i}, \mathscr{N}_{i}, \lambda_{i}\right)$ are isomorphic on $U \times U$. Let $(\mu, \nu):\left(\mathscr{M}_{2}, \mathscr{N}_{2}, \lambda_{2}\right) \rightarrow$ $\left(\mathscr{M}_{1}, \mathscr{N}_{1}, \lambda_{1}\right)$ be such an isomorphism.

Let $p_{i j}: U \times U \times U \rightarrow U \times U$ be the projection $\left(u_{1}, u_{2}, u_{3}\right) \rightarrow$ $\left(u_{i}, u_{j}\right)$ and for any sheaf of $\mathcal{O}_{U \times U}$ modules $\mathscr{L}$ let $r_{i j}: \Gamma(V, \mathscr{L}) \rightarrow$ $\Gamma\left(p_{i j}^{-1}(V), p_{i j}^{*} \mathscr{L}\right)$ be the corresponding map on sections. Consider the morphism $\phi=\left(r_{13} \mu\right)^{-1} \circ\left(r_{12} u\right) \circ\left(r_{23} \mu\right)=d \mu$ obtained by composing

$$
\begin{aligned}
p_{23}^{*} p_{2}^{*} \mathscr{M}^{r_{23} \mu} & \rightarrow p_{23}^{*} p_{13}^{*} p_{1}^{*} \mathscr{M} \simeq p_{12}^{*} p_{2}^{*} \mathscr{M}^{r_{12} \mu} \rightarrow p_{12}^{*} p_{1}^{*} \mathscr{M} \\
& \simeq p_{13}^{*} p_{1}^{*} \mathscr{M}^{\left(r_{13} \mu\right)^{-1}} \rightarrow p_{13}^{*} p_{2}^{*} \mathscr{M} \simeq p_{23}^{*} p_{2}^{*} \mathscr{M}
\end{aligned}
$$

and the analogous morphism $\phi^{\prime}=\left(r_{13} \nu\right)^{-1} \circ\left(r_{12} \nu\right) \circ\left(r_{23} \nu\right)=d \nu$ for $\nu$. Then the pair $\left(\phi, \phi^{\prime}\right)$ is an automorphism of the data $\left(p_{23}^{*} p_{2}^{*} \mathscr{M}\right.$, $\left.p_{23}^{*} p_{2}^{*} \mathscr{N}, p_{23}^{*} p_{2}^{*} \lambda\right)$ preserving the isomorphism of the resulting elementary algebra with $p_{23}^{*} p_{2}^{*} \mathscr{A}$. Hence, by Lemma $2.4, \phi \in \Gamma\left(U \times U \times U, \mathbf{G}_{m}\right)$ and $\phi^{\prime}=\phi^{-1}$. Clearly,

$$
d \phi=\left(r_{123} \phi\right)^{-1}\left(r_{124} \phi\right)\left(r_{134} \phi\right)^{-1}\left(r_{234} \phi\right)=1
$$

and so $\phi$ is a 2-cocycle for étale Čech cohomology (cf. [10], §III.2). If $\phi=d \gamma$ for some $\gamma \in \Gamma\left(U \times U, \mathbf{G}_{m}\right)$, then $\gamma^{-1} \mu$ and $\gamma \nu$ satisfy the cocycle condition and by descent theory $([10], 1.2 .22)$ we conclude that $(\mathscr{M}, \mathscr{N}, \lambda)$ 
has the form $\left(f^{*} \mathscr{M}_{0}, f^{*} \mathscr{N}_{0}, f^{*} \lambda_{0}\right)$ for data $\left(\mathscr{M}_{0}, \mathscr{N}_{0}, \lambda_{0}\right)$ on $X$ for which $\mathscr{A} \simeq \mathscr{M}_{0} \otimes^{\lambda_{0}} \mathscr{N}_{0}$. Thus, $\delta[\mathscr{A}]=[\phi]$ defines an injection $\delta$ from $\tilde{B}(X)$ to $H^{2}\left(X_{\text {et }}, \mathbf{G}_{m}\right)$.

The map $\delta$ constructed in this way is compatible with the one constructed using Giraud's machinery of gerbes. This follows from the results of $\S I V, 3.5$ of [7].

3. Surjectivity of $\delta$. Our proof that (under reasonable hypotheses) the map $\delta$ is surjective amounts to working backwards in the construction following Proposition 2.5. That is, given a Čech cocycle $\phi$ for an étale cover $U \rightarrow X$, we will (after possibly replacing $U \rightarrow X$ with a refinement) construct $\mathcal{O}_{U}$ modules $\mathscr{M}, \mathscr{N}$ and a surjective $\lambda: \mathscr{N} \otimes \mathscr{M} \rightarrow \mathcal{O}_{U}$. We do this in such a way that if $\mathscr{M}_{i}=p_{i}^{*} \mathscr{M}, \mathscr{N}_{i}=p_{i}^{*} \mathscr{N}, \lambda_{l}=p_{i}^{*} \lambda$ then there is an isomorphism $(\mu, \nu):\left(\mathscr{M}_{2}, \mathscr{N}_{2}, \lambda_{2}\right) \rightarrow\left(\mathscr{M}_{1}, \mathscr{N}_{1}, \lambda_{1}\right)$ such that $\mu \otimes \nu$ : $\mathscr{M}_{2} \otimes^{\lambda_{2}} \mathscr{N}_{2} \rightarrow \mathscr{M}_{1} \otimes^{\lambda_{1}} \mathscr{N}_{1}$ satisfies $d(\mu \otimes \nu)=1$ so that $\mathscr{M} \otimes^{\lambda} \mathscr{N}$ descends to a central separable $\mathcal{O}_{X}$ algebra $A$, but $d(\mu, \nu)=\left(\phi, \phi^{-1}\right)$ so that $\phi$ represents the obstruction to the descent of the data $(\mathscr{M}, \mathscr{N}, \lambda)$. By the construction following Proposition 2.5, we will then have $\delta[\mathscr{A}]=[\phi]$.

Our construction of $\mathscr{M}, \mathscr{N}$, and $\lambda$ depends on certain properties of the trace. We begin this section with a discussion of these properties.

Let $R$ be a ring and $S$ a commutative $R$-algebra (possibly without identity). The left action of $S$ on itself determines an algebra morphism $S \rightarrow \operatorname{End}_{R}(S)$. If $S$ is free and finite as an $R$-module, then $\operatorname{End}_{R}(S) \simeq$ $S \otimes_{R} S^{*}$ and, hence, the trace $\operatorname{tr}: \operatorname{End}_{R}(S) \rightarrow R$ is defined. On composing this with $S \rightarrow \operatorname{End}_{R}(S)$ we get an $R$-morphism $\tau: S \rightarrow R$. This behaves well under base change. That is, if $R \rightarrow R^{\prime}$ is a ring homomorphism, then the trace $\tau^{\prime}$ defined as above for $S \otimes_{R} R^{\prime}$ as an $R^{\prime}$-algebra is just $\tau \otimes 1$, where $\tau$ is the trace for $S$.

Now if $S$ is an $R$-algebra but is not free and finite, then there may not be a natural trace $S \rightarrow R$. In the case where $S$ is quasi-finite over $R$ it is, however, easy to decide what we should mean by a trace when such a thing exists. For $S$ to be quasi-finite over $R$ means that for each prime ideal $p \in \operatorname{Spec}(R), S \otimes_{R} k_{p}$ is a finite $k_{p}$ algebra, where $k_{p}$ is the residual field at $p$ for $R$. We shall say that an $R$-morphism $\tau: S \rightarrow R$ is a trace for $S$ if $\tau \otimes 1: S \otimes_{R} k_{p} \rightarrow k_{p}$ is the standard trace on $S \otimes_{R} k_{p}$ for each $p \in \operatorname{Spec}(R)$. Note that any two trace morphisms will differ by a map into the nilradical of $R$ and so, if a trace exists for $S$ and $R$ is reduced, the trace is unique.

Note that if $T$ is a subalgebra of an $R$-algebra $S$ then a trace for $S$ does not necessarily restrict to a trace for $T$ due to multiplicities that may 
occur in the action of $T$ on $S$ that don't occur in the action of $T$ on itself. However, if $T$ is an ideal of $S$ this does not happen.

LEMMA 3.1. Let $S$ be a quasi-finite $R$-algebra with a trace $\tau: S \rightarrow R$. Then $\left.\tau\right|_{T}$ is a trace for $T$ whenever $T$ is an ideal of $S$.

Proof. Let $p$ be a prime ideal of $R, i: T \rightarrow S$ the inclusion, and $K$ and $L$ the kernel and cokernel, respectively, of the induced map $i \otimes \mathrm{id}$ : $T \otimes_{R} k_{p} \rightarrow S \otimes_{R} k_{p}$. If we can show that each $t \in T$ acts trivially on both $K$ and $L$ then we are done, since this implies $t$ has the same trace acting on the vector space $T \otimes_{R} k_{p}$ as it does acting on $S \otimes_{R} k_{p}$.

That $t$ acts trivially on $L$ is obvious since $t S \subset T$. The action of $t$ on $T$ factors as $T \stackrel{i}{\rightarrow} S \stackrel{t}{\rightarrow} T$, and since $K$ is $\operatorname{ker}(i \otimes$ id $)$ it is clear that $t$ acts as zero on $K$ also.

Now let $f: Y \rightarrow X$ be a quasi-finite morphism of schemes. If $\mathscr{I} \subset \boldsymbol{\theta}_{Y}$ is a coherent sheaf of ideals, then an $\mathcal{O}_{X}$ module homomorphism $\tau$ : $f_{*} \mathscr{I} \rightarrow \mathcal{O}_{X}$ will be called a trace if for each $x \in X, \tau_{x}:\left(f_{*} \mathscr{I}\right)_{x} \rightarrow \mathcal{O}_{x}$ is a trace in the above sense. Again, if $X$ is reduced then a trace is unique when one exists. Also, by Lemma 3.1, the restriction of a trace to a smaller ideal sheaf is still a trace.

Definition 3.2. Let $f: U \rightarrow X$ be a morphism of Noetherian schemes. We shall say $f$ is of trace type if there is a factorization $U \stackrel{i}{\rightarrow} Y \stackrel{g}{\rightarrow} X$ of $f$, with $i$ an open immersion and $g$ finite and a coherent ideal sheaf $\mathscr{I} \subset \mathcal{O}_{Y}$ such that $V(\mathscr{I})=Y \backslash U$ and $\mathscr{I}$ has a trace $\tau: g_{*} \mathscr{I} \rightarrow \mathcal{O}_{X}$.

Here $V(\mathscr{I})$ is the support of $\mathcal{O}_{Y} \backslash \mathscr{I}$ or, in other words, the set of $y \in Y$ such that $\mathscr{I}_{y}$ is a proper ideal of $\mathcal{O}_{y}$ (see [9], II.5.9).

LEMMA 3.3. (a) If $f: U \rightarrow X$ is of trace type then so is its composition with an open immersion $V \rightarrow U$;

(b) If $f: U \rightarrow V$ is of trace type and $V \stackrel{k}{\rightarrow} X$ is an open immersion, then $k \circ f$ is also of trace type.

Proof. (a) Let $U \rightarrow Y \rightarrow X$ be the factorization and $\mathscr{I} \subset \mathcal{O}_{Y}$ the ideal of Definition 3.2. If $\mathscr{I}_{Y \backslash V}$ is the ideal sheaf of $Y \backslash V$, then $\mathscr{I}_{Y} \cap \mathscr{I}_{Y \backslash V}$ is an ideal sheaf with variety $Y \backslash V$ and is an ideal in $\mathscr{I}$. Thus, it has a trace by Lemma 3.3 and $V \rightarrow X$ is of trace type.

(b) Let $U \stackrel{i}{\rightarrow} W \stackrel{g}{\rightarrow} V$ be the factorization and $\mathscr{I} \subset \mathcal{O}_{W}$ the ideal of Definition 3.2. The composition $k \circ g$ : $W \rightarrow X$ is separated and quasifinite and so, by Zariski's main theorem ([10], I.1.8.), it factors as 
$W \stackrel{i}{\rightarrow} Y \stackrel{h}{\rightarrow} X$ with $j$ an open immersion and $h$ finite. Thus, we have the following commutative diagram:

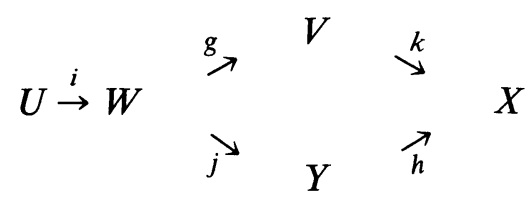

Now $\mathscr{I} \subset \mathscr{I}_{W \backslash U} \subset\left(\mathscr{I}_{Y \backslash U}\right) \mid W$ and so $\mathscr{I}$ extends to a coherent subsheaf $\mathscr{J}$ of $\mathscr{I}_{Y \backslash U}$ ([9], II ex. 5.15(d)). Necessarily, $\mathscr{J}$ is an ideal sheaf in $\mathcal{O}_{Y}$ with variety $Y \backslash U$.

We have the map $\mathscr{J} \rightarrow j_{*} j^{*} \mathscr{J}=j_{*} \mathscr{I}$, given by restriiction of sections from $Y$ to $W$, and, hence, a map

$$
h_{*} \mathscr{J} \rightarrow h_{*} j_{*} \mathscr{I}=k_{*} g_{*} \mathscr{I} \text {. }
$$

Also, the trace $\tau: g_{*} \mathscr{I} \rightarrow \mathcal{O}_{V}$ induces a map

$$
k_{*} g_{*} \mathscr{I} \rightarrow k_{*} \mathcal{O}_{V}
$$

and on composing we obtain a map

$$
\tau_{1}: h_{*} \mathscr{J} \rightarrow k_{*} \mathcal{O}_{V}
$$

Since $h$ is finite, $h_{*} \mathscr{J}$ is coherent ([9], II. ex 5.5) and, hence, so is its image under $\tau_{1}$ in the quasi-coherent module $h_{*} \mathcal{O}_{V}$.

Let $\mathscr{K}$ be the kernel of the natural map $\mathscr{O}_{X} \rightarrow k_{*} K^{*} \mathcal{O}_{X}=k_{*} \mathcal{O}_{V}$. Then $\mathscr{K}$ is a coherent ideal sheaf in $\mathcal{O}_{X}$ supported on $X \backslash V$. Thus, it is killed by some power of $\mathscr{I}_{X \backslash V}$ (this is true locally by II. 5.3 of [9] and the global result follows from quasicompactness of $X$ ). The Artin-Rees Lemma ([3], Cor. 10.10) then implies that $\left(\mathscr{I}_{X \backslash U}\right)^{n} \cap \mathscr{K}=\varnothing$ for some $n$, and we may regard $\left(\mathscr{I}_{X \backslash U}\right)^{n}$ as an $\mathcal{O}_{X}$ submodule of $k_{*} \mathcal{O}_{V}$. We may then consider the quotient sheaf $\tau_{1} h_{*} \mathscr{K} / \tau_{1} h_{*} \mathscr{L} \cap\left(\mathscr{J}_{X \backslash V}\right)^{n}$. This is coherent and supported in $X \backslash V$ and, hence, is killed by some power of $\mathscr{I}_{X \backslash V}$. In other words, for some $m$

$$
\left(\mathscr{I}_{X \backslash V}\right)^{m} \tau_{1} h_{*} \mathscr{J} \subset\left(\mathscr{I}_{X \backslash V}\right)^{n} .
$$

On replacing $\mathscr{J}$ with its product with $\left(h^{*} \mathscr{I}_{X \backslash V}\right)^{m}$, we obtain an ideal sheaf $\mathscr{G} \subset \mathcal{O}_{Y}$ such that $\tau_{1} h_{*} \mathscr{G} \subset\left(\mathscr{I}_{X \backslash V}\right)^{n} \subset \mathcal{O}_{X}$. Note that $V(\mathscr{G})=Y \backslash U$ and that $\tau_{1}$ is, in fact, a trace. This is obvious over points of $V$ (since $\tau$ was a trace). Over a point $x \in X \backslash V,\left(h_{*} \mathscr{G}\right) \otimes k_{x}$ is an ideal contained in every prime ideal of the finite $k_{x}$-algebra $\left(h_{*} \mathcal{O}_{Y}\right) \otimes k_{x}$ and is, hence, nilpotent. This implies that the standard trace on $\left(h_{*} \mathscr{G}\right) \oplus k_{x}$ is zero, as is $\tau_{1} \otimes \mathrm{id}:\left(h_{*} \mathscr{G}\right) \otimes k_{x} \rightarrow k_{x}$. 
LEMMA 3.4. let $f: U \rightarrow X$ be an étale cover of finite type of a Noetherian scheme $X$. Then there is an étale cover $g: \tilde{U} \rightarrow X$ of trace type which factors through $f$.

Proof. It follows from [10], I.3.14, that there is a finite cover $\left\{U_{i}\right\}$ of $U$ by open affine neighborhoods such that each $\left.f\right|_{U_{i}}$ factors as $U_{i} \rightarrow$ $W_{i} \stackrel{h_{i}}{\rightarrow} V_{i} \rightarrow X$ with $U_{i} \rightarrow W_{i}$ and $V_{i} \rightarrow X$ open immersions and $h_{i}$ finite and surjective with $\left(h_{i}\right)_{*} \mathcal{O}_{W_{i}}$ free over $\mathcal{O}_{V_{i}}$. Clearly, $h_{i}$ is of trace type (with $W_{i} \stackrel{\text { id }}{\rightarrow} W_{i} \stackrel{h_{i}}{\rightarrow} V_{i}$ as factorization and $\mathcal{O}_{W_{i}}$ the coherent ideal sheaf) and so, by Lemma 3.3, the composition $U_{i} \rightarrow X$ is trace type as well. If we let $\tilde{U}$ be the disjoint union of the $U_{i}$ and $g: \tilde{U} \rightarrow X$ the morphism such that $\left.g\right|_{U_{t}}=\left.f\right|_{U_{t}}$, then $g$ is clearly an étale cover of trace type which factors through $f$.

LEMMA 3.5. Let $f: U \rightarrow X$ be an étale cover of trace type with factorization $U \stackrel{i}{\rightarrow} Y \stackrel{g}{\rightarrow} X$, ideal sheaf $\mathscr{I} \subset \mathcal{O}_{Y}$, and trace $\tau$. Then $\tau$ : $\left(g_{*} \mathscr{I}\right) \rightarrow \mathcal{O}_{X}$ is surjective.

Proof. Given $x \in X, \tau:\left(g_{*} \mathscr{I}\right)_{x} \rightarrow \mathcal{O}_{x}$ fails to be surjective if and only if its image lies in the maximal ideal of $\mathcal{O}_{x}$. This happens if and only if $\tau \otimes$ id: $\left(g_{*} \mathscr{I}\right) \otimes k_{x} \rightarrow k_{x}$ is zero. However, since $g$ is finite, $\left(g_{*} \mathcal{O}_{Y}\right) \otimes k_{x}$ is the direct sum of the finite local algebras $\mathcal{O}_{y} \otimes_{\mathcal{O}_{x}} k_{x}$ for $y \in g^{-1}(x)$. Since $U \rightarrow X$ is an étale cover, $g^{-1}(x) \cap U \neq \varnothing$ and for $y$ in this set, $\mathcal{O}_{y} \otimes_{\mathcal{O}_{x}} k_{x}$ is a finite separable field extension of $k_{x}$ ([10], I.§3). The standard trace is therefore non-degenerate on $\mathcal{O}_{y} \otimes_{\mathcal{O}_{x}} k_{x}$ by Proposition I.3.1 of [10]. Since $\tau \otimes \mathrm{id}:\left(g_{*} \mathcal{O}_{Y}\right) \otimes k_{x} \rightarrow k_{x}$ agrees with the standard trace, it cannot be zero.

THEOREM 3.6. Let $X$ be a Noetherian scheme in which every finite set is contained in an affine set. Then $\delta: \tilde{B}(X) \rightarrow H^{2}\left(X_{\mathrm{et}}, \mathbf{G}_{m}\right)$ is an isomorphism.

Proof. We must prove that $\delta$ is surjective. The hypotheses are sufficient for us to identify $H^{2}\left(X_{\mathrm{et}}, \mathbf{G}_{m}\right)$ with the corresponding Čech cohomology group ([10], III. 2.17.) Also, it follows from Lemma 3.4 that every class in $H^{2}\left(X_{\mathrm{et}}, \mathbf{G}_{m}\right)$ arises from a Čech cocycle $\phi \in$ $\Gamma\left(U \times U \times U, \mathbf{G}_{m}\right)$ for an étale cover $f: U \rightarrow X$ which is of trace type.

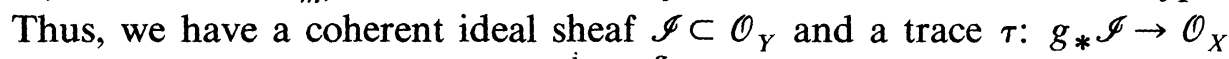
associated with a factorization $U \stackrel{i}{\rightarrow} \mathrm{Y} \stackrel{g}{\rightarrow} X$ as in Definition 3.2. 
We would like, via restriction to $U\left(\mathscr{I} \rightarrow i_{*} i * \mathscr{I}=i_{*} \mathcal{O}_{U}\right)$ to regard $\mathscr{I}$ as a subsheaf of $i_{*} \mathcal{O}_{U}$. However, $\mathscr{I} \rightarrow i_{*} \mathcal{O}_{U}$ may have non-zero kernel. This would be a coherent sheaf supported on $Y \backslash U$ and, hence, would be killed by a power of $\mathscr{I}_{Y \backslash U}$. If we replace $\mathscr{I}$ by this power of $\mathscr{I}_{Y \backslash U}$ times $\mathscr{I}$ we will have a coherent ideal sheaf which does inject into $i_{*} \mathcal{O}_{U}$. Thus, we may as well assume that $\mathscr{I}$ has this property and regard it as a subsheaf of $i_{*} \mathcal{O}_{U}$.

Let $U^{k}=U \times U \times \cdots \times U$ denote the $k$-fold fiber product of $U$ over $X$ and $Y \times U^{k}$ the fiber product of $Y$ with $U^{k}$ over $X$. We set

$$
\mathscr{K}=i_{*} \mathcal{O}_{U},
$$

and then extend the coherent sheaves $\mathscr{I} \subset \mathscr{K}$ on $Y$ to be sheaves on the étale site over $Y$. That is, for any étale map $h: V \rightarrow y$ we set $\mathscr{K}_{V}=h^{*} \mathscr{K}$ and $\mathscr{I}_{V}=h^{*} \mathscr{I}$. In particular, $\mathscr{K}_{Y \times U} k$ has this form where $h$ is the étale map $Y \times U^{k} \rightarrow Y$ obtained from base extension of $U^{k} \rightarrow X$ by $Y$ ([10], I.3.3(c)). However, we also have

$$
\mathscr{K}_{Y \times U^{k}}=i_{*} \mathcal{O}_{U^{k+1}}
$$

for the inclusion $i: U^{k+1} \rightarrow Y \times U^{k}$. This follows from III.9.3 of [9] applied to the square

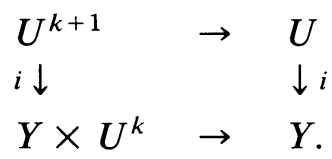

As a result, we may regard the cocycle $\phi \in \Gamma\left(U^{3}, \mathbf{G}_{m}\right)$ to be an invertible section of the sheaf of rings $\mathscr{K}_{Y \times U \times U}$.

We define sheaves $\mathscr{E}$ and $\mathscr{F}$ on $Y \times U$ as follows: Let $p_{0 i}: Y \times U \times U$ $\rightarrow Y \times U$ be defined by $p_{0 i}\left(y, u_{1}, u_{2}\right)=\left(y, u_{i}\right)$. Then we set

$$
\begin{aligned}
& \mathscr{E}=\mathscr{I}_{Y \times U} \cap\left(p_{02}\right)_{*} \phi^{-1} \mathscr{I}_{Y \times U \times U} \\
& \mathscr{F}=\mathscr{I}_{Y \times U} \cap\left(p_{02}\right)_{*} \phi \mathscr{I}_{Y \times U \times U}
\end{aligned}
$$

Here, $\phi^{-1} \mathscr{I}_{Y \times U \times U} \subset \mathscr{K}_{Y \times U \times U}$,

$$
\mathscr{I}_{Y \times U} \subset \mathscr{K}_{Y \times U} \subset\left(p_{02}\right)_{*}\left(p_{02}^{*}\right) \mathscr{K}_{Y \times U}=\left(p_{02}\right)_{*} \mathscr{K}_{Y \times U \times U},
$$

and so the intersection in the definition make sense. Note that $\mathscr{E}$ and $\mathscr{F}$ are coherent sheaves of ideals on $Y \times U$, are contained in $\mathscr{I}_{Y \times U}$, and satisfy $V(\mathscr{E})=V(\mathscr{F})=Y \times U \backslash U \times U$.

Now to say that an element of $\left(\mathscr{I}_{Y \times U}\right)_{(y, u)}$ belongs to the stalk of $\left(p_{02}\right)_{*} \phi^{-1} \mathscr{I}_{Y \times U \times U}$ at $(y, u)$ is the same as saying its lift to a section of $\mathscr{I}_{Y \times U \times U}$ over $p_{02}^{-1}(y, u)$ belongs to the sections of $\phi^{-1} \mathscr{I}_{Y \times U \times U}$. Thus, we 
have

$$
\phi p_{02}^{*} \mathscr{E} \subset \mathscr{I}_{Y \times U \times U} .
$$

We also need to know how $\mathscr{E}$ and $\mathscr{F}$ lift if we take fiber product with another copy of $U$. For this, we consider the commutative diagrams

$$
\begin{aligned}
& Y \times U^{3} \stackrel{p_{023}}{\rightarrow} Y \times U^{2} \quad Y \times U^{3} \stackrel{p_{023}}{\rightarrow} Y \times U^{2} \\
& \begin{array}{cccccc}
\downarrow p_{012} & & \downarrow p_{02} & \downarrow p_{013} & & \downarrow p_{02} \\
Y \times U^{2} & \stackrel{p_{01}}{\rightarrow} & Y \times U & Y \times U^{2} & \stackrel{p_{02}}{\rightarrow} & Y \times U
\end{array}
\end{aligned}
$$

where $p_{o l j}: Y \times U^{3} \rightarrow Y \times U^{2}$ is given by $p_{o i j}\left(y, u_{1}, u_{2}, u_{3}\right)=\left(y, u_{l}, u_{j}\right)$. If we put $\phi^{-1} \mathscr{I}_{Y \times U \times U}$ on the upper right hand corner of each diagram and apply III.9.3. of [10], we conclude that

$$
\begin{aligned}
& p_{01}^{*} \mathscr{E}=\mathscr{I}_{Y \times U \times U} \cap\left(p_{023}\right)_{*}\left(p_{012}^{*} \phi^{-1}\right) \mathscr{I}_{Y \times U^{3}} \\
& p_{02}^{*} \mathscr{E}=\mathscr{I}_{Y \times U \times U} \cap\left(p_{023}\right)_{*}\left(p_{013}^{*} \phi^{-1}\right) \mathscr{I}_{Y \times U^{3}} .
\end{aligned}
$$

The condition that $\phi$ be a cocycle is

$$
\left(p_{123}^{*} \phi\right)\left(p_{023}^{*} \phi\right)^{-1}\left(p_{013}^{*} \phi\right)\left(p_{012}^{*} \phi\right)^{-1}=1
$$

where $p_{123}\left(y, u_{1}, u_{2}, u_{3}\right)=\left(u_{1}, u_{2}, u_{3}\right)$. Since $p_{123}^{*} \phi$ does not involve the $Y$ variable, it maps $\mathscr{I}_{Y \times U^{3}}$ into itself and so (3) and (4) yield

$$
\begin{aligned}
\phi p_{02}^{*} \mathscr{E} & \subset \phi\left(p_{023}\right)_{*}\left(p_{023}^{*} \phi^{-1}\right) \mathscr{I}_{Y \times U^{3}}=\left(p_{023}\right)_{*}\left(p_{023}^{*} \phi\right)\left(p_{013}^{*} \phi\right)^{-1} \mathscr{I}_{Y \times U^{3}} \\
& =\left(p_{023}\right)_{*}\left(p_{012}^{*} \phi\right)^{-1} \mathscr{I}_{Y \times U^{3}} .
\end{aligned}
$$

Thus, (2), (3), and (5) imply that

$$
\phi\left(p_{02}\right)^{*} \mathscr{E} \subset\left(p_{01}\right)^{*} \mathscr{E}
$$

and, by symmetry, that $\phi^{-1}\left(p_{01}\right)^{*} \mathscr{E} \subset\left(p_{02}\right) * \mathscr{E}$. Thus, $\phi$ defines an isomorphism $p_{02}^{*} \mathscr{E} \rightarrow p_{01}^{*} \mathscr{E}$. The same argument, applied to $\mathscr{F}$, shows that $\phi^{-1}$ defines an isomorphism $p_{02}^{*} \mathscr{F} \rightarrow p_{01}^{*} \mathscr{F}$.

With $p_{1}: Y \times U \rightarrow U$ the projection, we now set

$$
\mathscr{M}=\left(p_{1}\right)_{*} \mathscr{E}, \quad \mathscr{N}=\left(p_{1}\right)_{*} \mathscr{F}
$$

and note that since $p_{i}: Y \times U \rightarrow U$ is finite ([10], I.1.3). $\mathscr{M}$ and $\mathscr{N}$ are coherent sheaves on $U$ ([9], II.ex.5.5). From the above, we have isomorphisms

$$
\mu: p_{2}^{*} \mathscr{M} \rightarrow p_{1}^{*} \mathscr{M}, \quad \nu: p_{2}^{*} \mathscr{N} \rightarrow p_{1}^{*} \mathscr{N}
$$


determined by $\phi$ and $\phi^{-1}$, where $p_{i}: U \times U \rightarrow U$ is given by $p_{i}\left(u_{1}, u_{2}\right)=$ $u_{i}$.

Since $\mathscr{E} \mathscr{F}$ is an ideal sheaf in $\mathscr{I}_{Y \times U}$ it has a trace $\tau: \mathscr{E} \mathscr{F} \rightarrow \mathscr{O}_{U}$. This is obtained from the trace for $\mathscr{I}_{Y}$ by the base extension $U \rightarrow X$ and then restriction to $\mathscr{E} \mathscr{F}$. Clearly, a base extension of a trace is a trace. Since $V(\mathscr{E} \mathscr{F})=Y \times U \backslash U \times U$ and $U \times U \rightarrow U$ is an étale cover, Lemma 3.5 implies that $\tau$ is surjective. We use $\tau$ to define a morphism $\lambda: \mathscr{N} \otimes \mathscr{M} \rightarrow$ $\mathcal{O}_{U}$ by $n \otimes m \rightarrow \tau(n m)$ (using $\left.\left(p_{1}\right)_{*} \mathscr{E} \mathscr{F}=\left(\left(p_{1}\right)_{*} \mathscr{E}\right)\left(p_{1}\right)_{*} \mathscr{F}\right)$.

At this point, we have data $(\mathscr{M}, \mathscr{N}, \lambda)$ defining an elementary $\mathcal{O}_{U}$ algebra $\mathscr{M} \otimes_{\hat{O}_{U}}^{\lambda} \mathscr{N}$ over $U$. We also have an isomorphism of data $(\mu, \nu)$ : $\left(p_{2}^{*} \mathscr{M}, p_{2}^{*} \mathscr{N}, p_{2}^{*} \lambda\right) \rightarrow\left(p_{1}^{*} \mathscr{M}, p_{1}^{*} \mathcal{N}, p_{1}^{*} \lambda\right)$ over $U \times U$ and a short calculation using (4) pushed forward to $U \times U \times U$ shows that $d(\mu, \nu)=$ $\left(\phi, \phi^{-1}\right)$ (cf. the end of $\left.\S 2\right)$ on $U \times U \times U$. This implies that $d(\mu \otimes \nu)=1$ so that $\mathscr{M} \otimes^{\lambda} \mathscr{N}$ descends to be a central separable algebra $\mathscr{A}$ on $X([\mathbf{1 0}]$, I.2.22) with $f^{*} \mathscr{A}=\mathscr{M} \otimes^{\lambda} \mathscr{N}$. The obstruction to the descent of the data $(\mathscr{M}, \mathscr{N}, \lambda)$ is $\phi$ as in the second proof of Proposition 2.5. Therefore $\delta[\mathscr{A}]=[\phi]$.

4. Conclusions. The Noetherian hypothesis in Theorem 3.6 may be removed in a lot of important cases by using continuity results for $H^{2}\left(X_{\text {et }}, \mathbf{G}\right)$. While we have not proved that $\tilde{B}$ is a continuous functor, we did prove, for rings, the continuity of a closely related functor $\bar{B}$ in [12]. If $R$ is a ring then $\bar{B}(R)$ is the subgroup of $\tilde{B}(R)$ consisting of classes which contain a central separable algebra which is a finitely presented $R$-module (cf. [12], Proposition 6.5).

Corollary 4.1. If $R$ is any ring, then $\tilde{B}(R)=\bar{B}(R)$ and $\delta: \tilde{B}(R) \rightarrow$ $H^{2}\left(\operatorname{Spec}(R)_{\mathrm{et}}, \mathbf{G}_{m}\right)$ is an isomorphism.

Proof. We have injections $\bar{B}(R) \rightarrow \tilde{B}(R) \rightarrow H^{2}\left(\operatorname{Spec}(R)_{\mathrm{et}}, \mathbf{G}_{m}\right)$ for general $R$, both of which are isomorphisms for Noetherian rings (Theorem 3.6, and [12], Proposition 6.4). The continuity of $\bar{B}$ and $H^{2}$ implies these maps are isomorphisms for any ring $R([12]$, Proposition 6.5, and [2], exposé VII.5.7).

For any ring $R, B(R)$ is a torsion group ([10], IV.2.7) and, in fact, a recent result of Gabber shows that it is always exactly the torsion subgroup of $H^{2}\left(X_{\mathrm{et}}, \mathbf{G}_{m}\right)$ (cf. [6]). However, $\tilde{B}(R)$ need not be a torsion group. In fact, we proved in [12] that $\tilde{B}(C(\Delta)) \simeq H^{3}(\Delta, \mathbf{Z})$ if $\Delta$ is a compact Hausdorff space and $C(\Delta)$ is the algebra of continuous functions on $\Delta$. Since $C(\Delta)$ is a limit of quotients of polynomial algebras over $\mathbf{C}$ (as is any algebra over $\mathbf{C}$ ), we must have $\tilde{B}(R)$ non-torsion for some 
quotients of polynomial algebras. The same conclusion can be drawn from Grothendieck's example ([8], II.16). Similarly, $\tilde{B}(R)$ will be non-torsion for some examples of algebras of holomorphic functions on Stein compact sets (cf. [12], §6) since every Banach algebra is a limit of such algebras.

On the other hand, under mild regularity conditions, we have $\tilde{B}(R)=$ $B(R)$ as we shall see below. In fact, this happens for some Banach algebras and algebras of holomorphic functions where the underlying Hausdorff Gelfand spectrum has non-torsion $H^{3}(\cdot, \mathbf{Z})$.

We first prove the following proposition:

Proposition 4.2. If $R$ is a Henselian local ring then $\tilde{B}(R)=B(R)$.

Proof. That $H^{2}\left(X_{\mathrm{et}}, \mathbf{G}_{m}\right) \simeq B(R)$ for $X=\operatorname{Spec}(R)$, in this case, is well known and so we could just appeal to Proposition 2.5 (cf. [10], IV. Cor. 2.12). However, a direct proof is so simple, we include it as evidence of the utility of $\tilde{B}(R)$.

By Proposition 4.3 of [12] a central separable $R$-algebra $A$ which contains a non-degenerate idempotent $p$ is equivalent to $p A p$ which is an Azumaya algebra. If $M$ is the maximal ideal of $R$, then $A / M A$ is a central separable $R / M$-algebra by Proposition 2.7 of [12]. If we can show that this implies that $A / M a$ contains a nondegenerate idempotent, it will follow from the Henselian hypothesis (as in Proposition 4.9 of [12]) that the idempotent lifts to one in $A$ and, hence, that $A$ is equivalent to an Azumaya algebra.

To finish the proof, it suffices to prove that if $A$ is a central separable algebra over a field $R$ then $A$ contains a non-zero idempotent. We may assume $A$ is finite dimensional by Corollary 4.2 of [12]. Since $A^{2}=A, A$ is not nilpotent and, hence, contains a non-nilpotent element $b$. The sequence $A \subset b A b \subset b^{2} A b^{2} \subset \cdots \subset b^{n} A b^{n} \subset \cdots$ necessarily terminates by finite dimensionality. Thus, if $c=b^{k}$ for a sufficiently large $k$, then $c^{3} A c^{3}=c A c$, so that $c^{3} a c^{3}=c^{3}$ for some $a \in A$. This yields an idempotent $c^{3} a$ which is non-zero because $c^{3}$ is non-zero, and the proposition is proved.

If $R$ is a regular integral domain then the inclusion $R \rightarrow K$ of $R$ in its field of fractions induces an injection $H^{2}\left(\operatorname{Spec}(R)_{\mathrm{et}}, \mathbf{G}_{m}\right) \rightarrow$ $H^{2}\left(\operatorname{Spec}(K)_{\mathrm{et}}, \mathbf{G}_{m}\right)([\mathbf{1 0}]$, IV. Corollary 2.6). In view of Proposition 2.5, we have the same result for $\tilde{B}(\operatorname{Spec}(R))$ and, hence, by Proposition 4.2 we have

Proposition 4.3. If $R$ is a regular integral domain, then $\tilde{B}(\operatorname{Spec}(R))$ is a torsion group. 
This can be used to settle one issue raised in [12]. Let $\Delta$ be a Stein compact subset of a Stein manifold and let $\mathcal{O}(\Delta)$ denote the algebra of functions holomorphic in a neighborhood of $\Delta$. In [12] a homomorphism $\delta: \tilde{B}(\mathcal{O}(\Delta)) \rightarrow H^{3}(\Delta, \mathbf{Z})$ was constructed. It was shown to be injective when $\mathcal{O}(\Delta)$ is Noetherian and the question was raised as to its surjectivity (cf. [12], Propositions 6.4 and 6.6). It is now easy to see that it need not be surjective. In fact, under reasonably mild restrictions on $\Delta, \mathcal{O}(\Delta)$ is Noetherian and regular. It is Noetherian if $\Delta$ is analytic, i.e. defined by finitely many inequalities of the form $|f(z)| \leq k$ for $f$ holomorphic in a neighborhood of $\Delta$. It is regular if $\Delta$ is a Stein compact subset of $\mathbf{C}^{n}$ with the property that no open set $U$ in $\mathbf{C}^{n}$ intersects $\Delta$ in a set which is contained in a lower dimensional subvariety of $U$. This ensures that $M_{x} / M_{x}^{2}$ has dimension $n$, where $M_{x}$ is the maximal ideal at $x$ in $\mathcal{O}(\Delta)$. It also ensures that

$$
\begin{aligned}
(0) & \subset\left(z_{1}-x_{1}\right) \mathcal{O}(\Delta) \subset \cdots \subset\left(z_{1}-x_{1}\right) \cdots\left(z_{r}-x_{r}\right) \mathcal{O}(\Delta) \\
& \subset \cdots \subset M_{x}
\end{aligned}
$$

is a filtration of $M_{x}$ by distinct prime ideals and, hence, that $M_{x}$ has height $n$ as well. It follows that $\mathcal{O}(\Delta)$ is regular in this case (cf. [9], I.5.) It will be an integral domain if $\Delta$ is connected and then, by Proposition 4.3, $\tilde{B}(\mathcal{O}(\Delta))$ will be torsion. Of course, it is easy to arrange for all these hypotheses to be satisfied and still have $H^{3}(\Delta, \mathbf{Z})$ be non-torsion. For example, let $\Delta$ be $A^{3} \subset \mathbf{C}^{3}$ where $A \subset \mathbf{C}$ is a compact annulus.

The analogous question for Banach algebras was also raised in [12]. That is, let $R$ be a commutative Banach algebra over $\mathbf{C}$ and let $\Delta$ be its maximal ideal space with the Gelfand topology. Then we still have the homomorphism $\delta: \tilde{B}(R) \rightarrow H^{3}(\Delta, \mathbf{Z})$ (see [12], §7). It was proved in [4] that $\delta$ maps the usual Brauer group $B(R)$ onto the torsion subgroup of $H^{3}(\Delta, \mathbf{Z})$. We observed earlier that when $R=C(\Delta)$ the homomorphism $\delta$ maps $\tilde{B}(R)$ onto $H^{3}(\Delta, \mathbf{Z})$, and it is natural to ask whether $\delta$ is always surjective on $\tilde{B}(R)$. It is not, of course, as the following example shows. Let $A \subset A_{1} \subset \mathbf{C}$ be two compact annuli with $A$ contained in the interior of $A_{1}$. Let $R$ be the algebra of functions $A_{1}^{3} \rightarrow \mathrm{C}$ which are continuous on $A_{1}^{3}=\Delta_{1}$ and analytic on its interior (which is a neighborhood of $\Delta=A^{3}$ ). Then restriction defines a ring homomorphism $R \rightarrow \mathcal{O}(\Delta)$. We have a commutative diagram.

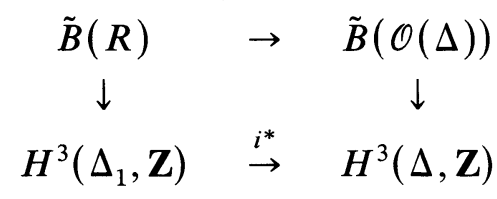


Here $i^{*}$ is an isomorphism and, in fact, $H^{3}\left(\Delta_{1}, \mathbf{Z}\right) \simeq H^{3}(\Delta, \mathbf{Z}) \simeq \mathbf{Z}$. Since the image of $\tilde{B}(\mathcal{O}(\Delta))$ in $H^{3}(\Delta, \mathbf{Z})$ must be torsion by the above, we have $\tilde{B}(R)=\tilde{B}(\mathcal{O}(\Delta))=(0)$ but $H^{3}(\Delta, \mathbf{Z}) \neq(0)$.

\section{REFERENCES}

[1] M. Artin, On the Joins of Hensel rings, Advances in Math. 7 (1971), 282-296.

[2] M. Artin, A. Grothendieck, and J. L. Verdier, (SGA4) Théorie des topos et cohomologie étale des Schémas (1963-64), Lecture Notes in Math. 269, 270, 305, Springer, Heidelberg, 1972-73.

[3] M. Atiyah and I. Macdonald, Introduction to Commutative Algebra, Addison-Wesley, Reading, Mass., 1969.

[4] I. Craw and S. Ross, Separable algebras over a commutative Banach algebra, Pacific J. Math., 104 (1982), 317-336.

[5] J. Dixmier, and A. Douady, Champs continus de'espaces Hilbertiens et $C^{*}$-algebras, Bull. Soc. Math. France, 91 (1963), 227-284.

[6] O. Gabber, Some Theorems on Azumaya algebras, Ph.D. Thesis, Harvard Univ., Cambridge, Mass., 1978.

[7] J. Giraud, Cohomologie Non Abéliennes, Springer, Heidelberg, 1971.

[8] A. Grothendieck, Le groupe de Brauer, I. Algébres de'Azumaya et interprétations diverses, II. Theorie cohomologique III. Exemples et compléments, in Dix Exposés sur la Cohomologie des Schémas, North Holland, Amsterdam, (1968), 46-188.

[9] R. Hartshorne, Algebraic Geometry, Springer, Heidelberg, 1977.

[10] J. S. Milne, Étale Cohomology, Princeton University Press, Princeton, 1980.

[11] I. Raeburn and J. L. Taylor, Continuous trace $C^{*}$-algebras with given Dixmier-Douady class, submitted for publication.

[12] J. L. Taylor, A bigger Brauer group, Pacific J. Math., 103 (1982), 163-203.

Received August 23, 1983. Research of the first author was partially supported by the Australian Research Grants Scheme. Research of the second author was supported by the National Science Foundation.

UNIVERSITY OF UTAH

Salt Lake City, UT 84112 



\section{PACIFIC JOURNAL OF MATHEMATICS EDITORS}

\author{
V. S. VARADARAJAN (Managing Editor) \\ University of California \\ Los Angeles, CA 90024 \\ Charles R. DePrima \\ California Institute of Technology \\ Pasadena, CA 91125 \\ R. FInN \\ Stanford University \\ Stanford, CA 94305
}

\section{HeRmanN FlaschKa \\ University of Arizona \\ Tucson, AZ 85721}

Ramesh A. Gangolli

University of Washington

Seattle, WA 98195

ROBION KIRBY

University of California

Berkeley, CA 94720

\section{C. MOORE}

University of California

Berkeley, CA 94720

\section{H. SAMELSON}

Stanford University

Stanford, CA 94305

HAROLD STARK

University of California, San Diego

La Jolla, CA 92093

\section{ASSOCIATE EDITORS}
R. ARENS
E. F. BECKENBACH
B. H. NeumanN
F. Wolf
K. Yoshida (1906-1982)

\section{SUPPORTING INSTITUTIONS}
CALIFORNIA INSTITUTE OF TECHNOLOGY

\author{
UNIVERSITY OF SOUTHERN CALIFORNIA \\ STANFORD UNIVERSITY \\ UNIVERSITY OF HAWAII \\ UNIVERSITY OF TOKYO \\ UNIVERSITY OF UTAH \\ WASHINGTON STATE UNIVERSITY \\ UNIVERSITY OF WASHINGTON
}
UNIVERSITY OF OREGON

\begin{abstract}
UNIVERSITY OF ARIZONA
UNIVERSITY OF BRITISH COLUMBIA

UNIVERSITY OF CALIFORNIA

MONTANA STATE UNIVERSITY

UNIVERSITY OF NEVADA, RENO

NEW MEXICO STATE UNIVERSITY

OREGON STATE UNIVERSITY
\end{abstract}

The Supporting Institutions listed above contribute to the cost of publication of this Journal, but they are not owners or publishers and have no responsibility for its content or policies.

Mathematical papers intended for publication in the Pacific Journal of Mathematics should be in typed form or offset-reproduced (not dittoed), double spaced with large margins. Please do not use built up fractions in the text of the manuscript. However, you may use them in the displayed equations. Underline Greek letters in red, German in green, and script in blue. The first paragraph must be capable of being used separately as a synopsis of the entire paper. In particular it should contain no bibliographic references. Please propose a heading for the odd numbered pages of less than 35 characters. Manuscripts, in triplicate, may be sent to any one of the editors. Please classify according to the scheme of Math. Reviews, Index to Vol. 39. Supply name and address of author to whom proofs should be sent. All other communications should be addressed to the managing editor, or Elaine Barth, University of California, Los Angeles, California 90024.

There are page-charges associated with articles appearing in the Pacific Journal of Mathematics. These charges are expected to be paid by the author's University, Government Agency or Company. If the author or authors do not have access to such Institutional support these charges are waived. Single authors will receive 50 free reprints; joint authors will receive a total of 100 free reprints. Additional copies may be obtained at cost in multiples of 50 .

The Pacific Journal of Mathematics is issued monthly as of January 1966. Regular subscription rate: $\$ 190.00$ a year (5 Vols., 10 issues). Special rate: $\$ 66.00$ a year to individual members of supporting institutions.

Subscriptions, orders for numbers issued in the last three calendar years, and changes of address should be sent to Pacific Journal of Mathematics, P.O. Box 969, Carmel Valley, CA 93924, U.S.A. Old back numbers obtainable from Kraus Periodicals Co., Route 100, Millwood, NY 10546.

The Pacific Journal of Mathematics at P.O. Box 969, Carmel Valley, CA 93924 (ISSN 0030-8730) publishes 5 volumes per year. Application to mail at Second-class postage rates is pending at Carmel Valley, California, and additional mailing offices. Postmaster: Send address changes to Pacific Journal of Mathematics, P.O. Box 969, Carmel Valley, CA 93924.

PUBLISHED BY PACIFIC JOURNAL OF MATHEMATICS, A NON-PROFIT CORPORATION

Copyright $\odot 1985$ by Pacific Journal of Mathematics 


\section{Pacific Journal of Mathematics}

Vol. 119, No. $2 \quad$ June, 1985

Mustafa Agah Akcoglu and Meira Falkowitz (Soshniak), A general local

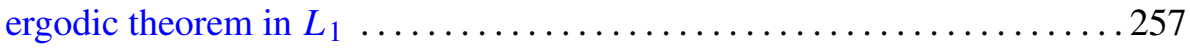

W. Wistar (William) Comfort and Lewis Chandlee Robertson,

Cardinality constraints for pseudocompact and for totally dense

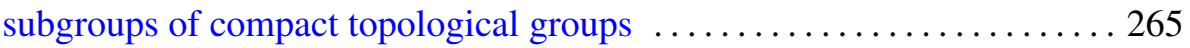

John Morse Delaurentis and Boris G. Pittel, Random permutations and

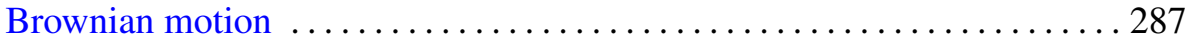

José Esteban Galé, Gel'fand theory in algebras of differentiable functions on Banach spaces ......................................... 303

Harry Gingold, On the location of zeroes of oscillatory solutions of

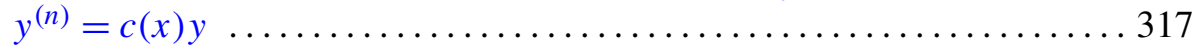

Kei Ji Izuchi, Zero sets of interpolating Blaschke products .............337

Mahesh Nerurkar, Ergodic continuous skew product actions of amenable

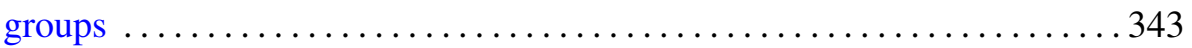

R. Owens, A maximal function characterization of a class of Hardy

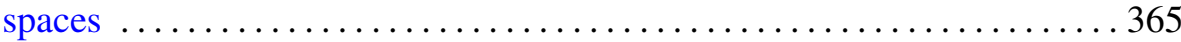

Judith Anne Packer, Point spectrum of ergodic abelian group actions and the corresponding group-measure factors $\ldots \ldots \ldots \ldots \ldots \ldots \ldots \ldots \ldots \ldots \ldots \ldots$

Judith Anne Packer, On the embedding of subalgebras corresponding to quotient actions in group-measure factors $\ldots \ldots \ldots \ldots \ldots \ldots \ldots \ldots . \ldots 4$

Iain Raeburn and Joseph L. Taylor, The bigger Brauer group and étale cohomology

David Rosen, The Diophantine equation $a x+b y=c$ in $Q(\sqrt{5})$ and other number fields

Mau-Hsiang Shih and Kok Keong Tan, Noncompact sets with convex sections

Lee Barlow Whitt, Codimension two isometric immersions between Euclidean spaces

Rodney Ian Yager, Iwasawa theory for the anticyclotomic extension 